\title{
Novel approach to hydroxy-group-containing porous organic polymers from bisphenol A
}

\author{
Tao Wang ${ }^{1,2}$, Yan-Chao Zhao ${ }^{1}$, Li-Min Zhang ${ }^{1}$, Yi Cui ${ }^{1}$, Chang-Shan Zhang ${ }^{2}$ \\ and Bao-Hang Han ${ }^{* 1}$
}

\section{Full Research Paper}

\section{Address:}

${ }^{1}$ CAS Key Laboratory of Nanosystem and Hierarchical Fabrication, CAS Center for Excellence in Nanoscience, National Center for Nanoscience and Technology, Beijing 100190, China and ${ }^{2}$ School of Chemical Engineering, Nanjing University of Science and Technology, Nanjing 210094, China

Email:

Bao-Hang Han * - hanbh@nanoctr.cn

* Corresponding author

Keywords:

bisphenol $\mathrm{A}$; carbon dioxide uptake; hydrogen storage;

$\mathrm{OH}$-containing; porous organic polymers

\author{
Beilstein J. Org. Chem. 2017, 13, 2131-2137. \\ doi:10.3762/bjoc.13.211
}

Received: 25 January 2017

Accepted: 20 September 2017

Published: 12 October 2017

This article is part of the Thematic Series "Organic porous materials".

Guest Editor: S. Bräse

(c) 2017 Wang et al.; licensee Beilstein-Institut. License and terms: see end of document.

\begin{abstract}
We successfully employed bisphenol A and several different formyl-containing monomers as useful building blocks to construct a series of hydroxy-group-containing porous organic polymers in a sealed tube at high temperature. Fourier transform infrared and

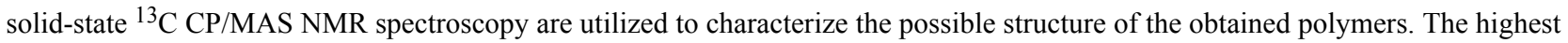
Brunauer-Emmet-Teller specific surface area of the phenolic-resin porous organic polymers (PPOPs) is estimated to be $920 \mathrm{~m}^{2} \mathrm{~g}^{-1}$. The PPOPs exhibit a highest carbon dioxide uptake (up to $15.0 \mathrm{wt} \%(273 \mathrm{~K})$ and $8.8 \mathrm{wt} \%(298 \mathrm{~K})$ at $1.0 \mathrm{bar}$ ), and possess moderate hydrogen storage capacities ranging from 1.28 to $1.04 \mathrm{wt} \%(77 \mathrm{~K})$ at 1.0 bar. Moreover, the highest uptake of methane for the PPOPs is measured as $4.3 \mathrm{wt} \%(273 \mathrm{~K})$ at $1.0 \mathrm{bar}$.
\end{abstract}

\section{Introduction}

Porous organic polymers standing out from kinds of porous materials such as zeolite, activated carbon, metal-organic frameworks [1,2], and covalent organic frameworks [3,4], with their prominent potential as heterogeneous catalysts [5-7], supports for catalysts [8,9], gas permeable membranes [10,11], and gas storage materials [12-14] have attracted much attentions from researchers all over the world as reviewed by Matyjaszewski
[15]. During the past years, a large amount of porous organic polymers (POPs) have been reported via Sonogashira-Hagihara coupling reaction [16], Suzuki-Miyaura chemistry [17], Yamato reaction [18], and self condensation of aromatic nitriles[19]. Although these methods can be used to construct POPs with high specific surface area values, these reactions are usually catalyzed by heavy and/or transition-metal catalysts, 
which are usually expensive and environmentally harmful. Furthermore, the majority of reagents used for the preparation of the aforementioned POPs are synthesized through multiple steps at high cost. New reactions using inexpensive and convenient raw materials with non-metallic catalysts, even no catalysts might show great advantages for construction of porous organic polymers. Our group has made much contribution to the exploitation of such reaction methodologies without any metallic catalysts [20-22].

We found that Bakelite-type chemistry is a reaction that can be catalyzed without any metal-containing catalysts and it is selected as an appropriate approach, spontaneously. Phenolic resins can be produced commercially using bases (ammonia and sodium hydroxide) or acids (hydrochloric acid and sulfuric acid) as catalysts via connecting phenolic molecules with formaldehyde or other aromatic aldehydes to form cross-linking structures through the simple Bakelite-type chemistry to obtain great number of polymers with different functionalities. Phloroglucinol has been utilized as a monomer by Kanatzidis and Katsoulidis $[23,24]$ to produce a series of porous polymers. In this contribution, bisphenol A (BPA) was employed as a novel polyphenol monomer instead of phloroglucinol. BPA is a commercially available industrial raw material, which is much cheaper than phloroglucinol. In addition, BPA might be stored more easily, compared with phloroglucinol that has a relatively high reactivity and can be oxidized in an ambient environment. Based on the aforementioned, BPA, to the best of our knowledge, may be a suitable candidate prior to other phenolic com- pounds such as phloroglucinol [23] and 1,5-dihydroxynaphthalene [24], which have been used for the preparation of porous materials.

Recently, Kanatzidis and Katsoulidis have reported a series of Bakelite-type porous organic polymers prepared in two steps. The mixture of reagents and solvent was pretreated at $70{ }^{\circ} \mathrm{C}$ and kept for $1 \mathrm{~h}$, followed by a high temperature treatment at $220^{\circ} \mathrm{C}$ for $96 \mathrm{~h} \mathrm{[25].} \mathrm{This} \mathrm{approach} \mathrm{is} \mathrm{involved} \mathrm{with} \mathrm{a} \mathrm{longer} \mathrm{reaction}$ time and a higher reaction temperature, which might cause a tremendous energy waste. Herein we provide an effective onestep approach to construct phenolic-resin porous organic polymers (PPOPs) from the reactions between BPA and different aldehydes using $p$-toluenesulfonic acid (TSA) as catalyst that has been proved to be a non-metallic acidic catalyst with high efficiency [26,27]. The materials exhibit Brunauer-Emmet-Teller (BET) specific surface area values ranging from 720 to $920 \mathrm{~m}^{2} \mathrm{~g}^{-1}$, and the highest carbon dioxide uptake is up to $15.0 \mathrm{wt} \%$ at $273 \mathrm{~K}$ and 1.0 bar. Meanwhile, the hydrogen and methane capacities are also investigated. Considering the gas adsorption properties, PPOPs may be a promising candidate for gas storage and separation materials.

\section{Results and Discussion}

Three multi-formyl compounds, i.e., two dialdehydes M1 and M2 [22] and one trialdehyde M3 [22] were employed to react with bisphenol A to produce phenolic-resin porous polymers PPOP-1-PPOP-3 (Scheme 1). It is well-known that the orthoand para-position of phenol are activated with negative charge

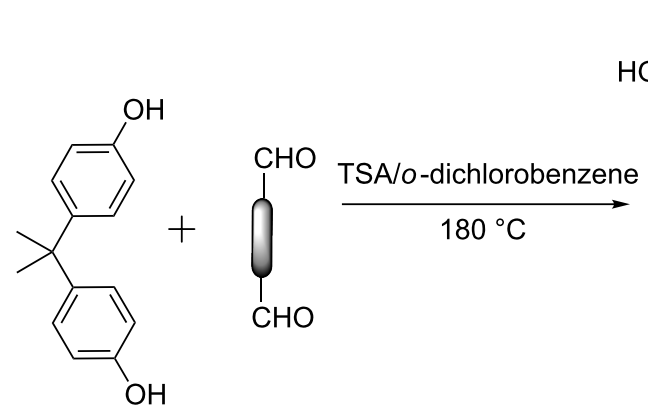<smiles>[Z]c1cc(C)c(O)c(C(CCCCC(c2cc(C)cc([Z])c2O)c2cc(C)cc(C)c2O)c2cc(C(C)(C)c3cc(C)c(O)c(C)c3)ccc2O)c1</smiles>

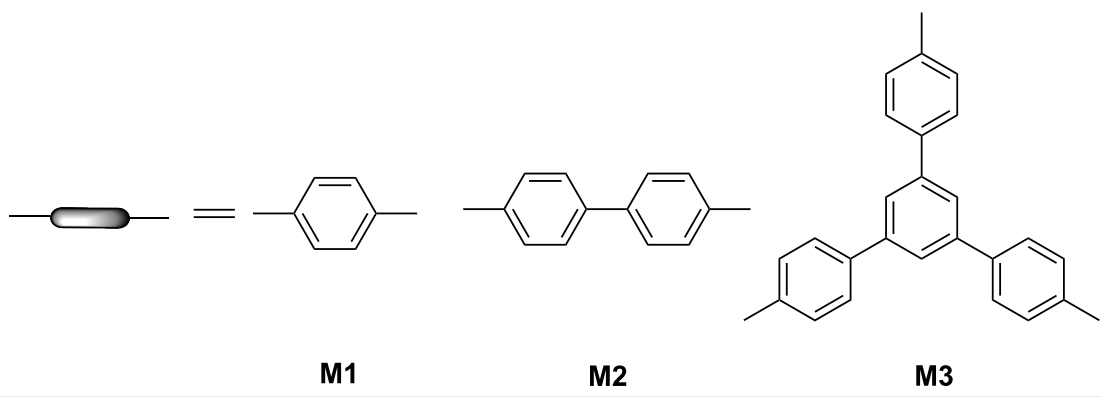

Scheme 1: Schematic representation of the possible structures of bisphenol-A-based porous organic polymers. 
for the electrophilic aromatic substitution in consequence of the electron-donating effect of the hydroxy group. The positively charged carbonyl group of the aldehyde could be attacked by the electron-rich phenyl ring, thus a carbonyl group can connect two phenol molecules by elimination of a water molecule. As a result, a cross-linked hydroxy-group-containing polymer is constructed ultimately. BPA and multi-formyl-containing compounds are suspended in $o$-dichlorobenzene, and TSA, as a catalyst, was then added into reaction system. After the reaction in a sealed tube at $180{ }^{\circ} \mathrm{C}$ for $72 \mathrm{~h}$, three polymers PPOP-1-PPOP-3 were obtained. The possible chemical structures of the obtained PPOPs are shown in the Scheme 1. All of the polymers are stable and insoluble in common organic solvents such as dichloromethane, ethanol, and acetone. Furthermore, the as-prepared materials exhibit a high thermal stability according to the results of TGA (Supporting Information File 1, Figure S1). There is a weight loss of about $5 \%$ up to $150{ }^{\circ} \mathrm{C}$, which is attributed to the evaporation of trapped solvent, carbon dioxide, or adsorbed water that could not be easily removed from the microporous structure of the polymers during the after-synthesis treatment and drying process. There is not any obvious thermal degradation for PPOPs until when heated up to $300{ }^{\circ} \mathrm{C}$.

According to the TGA result of BPA (Supporting Information File 1, Figure S2), the thermal degradation of BPA begins around $180^{\circ} \mathrm{C}$ and the evolved products are mainly phenol with one or two benzene rings from investigations of thermal degradation of bisphenol A polycarbonate [28]. Investigation of the changes from methyl groups and aldehyde groups between monomers and polymers is carried out by means of FTIR spectroscopy. The FTIR spectra displayed (Figure 1 and Supporting Information File 1, Figure S3) that signal at $2970 \mathrm{~cm}^{-1}$ arised from the stretching vibration of methyl groups shows quantitative changes between BPA and PPOPs, suggesting the degradation of BPA in $o$-dichlorobenzene and it is reported that the cleavage of methylene can be catalyzed under acidic or basic conditions [29,30]. $p$-Toluenesulfonic acid acted as an acid catalyst in this contribution and will promote the cleavage of BPA at high temperature. The broad absorption bands located at ca. $3500 \mathrm{~cm}^{-1}$ is attributed to the characteristic stretching vibration of hydroxy groups, which is consistent with the literature data [25]. The absorption peak at $1705 \mathrm{~cm}^{-1}$ assigned to the stretching vibration of carbonyl groups is significantly reduced in PPOPs, indicating that most of the aldehyde compounds are consumed.

Solid-state ${ }^{13} \mathrm{C}$ CP/MAS NMR spectroscopy was employed to characterize the structure of the polymers PPOPs. As shown in Figure 2 and Figure S4 (Supporting Information File 1), it can be found that the ${ }^{13} \mathrm{C}$ chemical shifts of these polymers are sim-

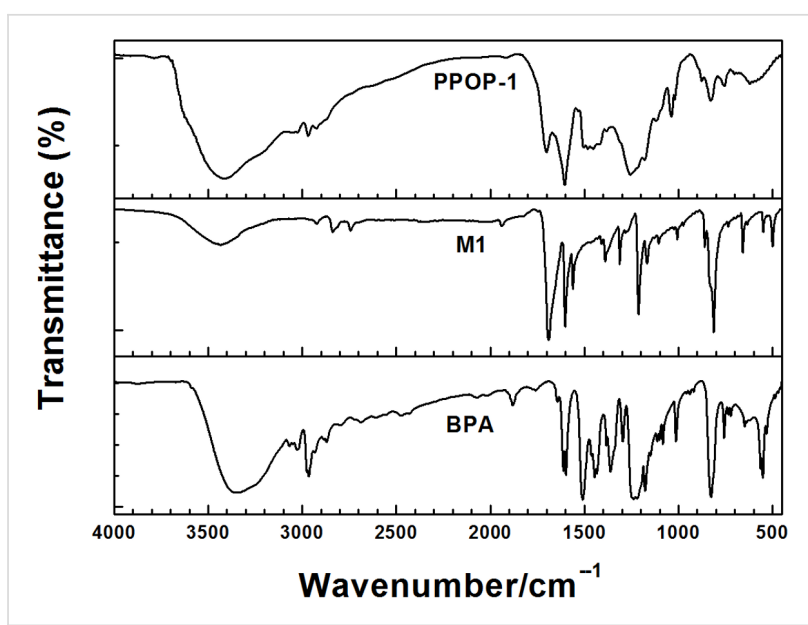

Figure 1: FTIR spectra of terephthalic aldehyde (M1), BPA, and PPOP-1.

ilar. It is reasonable considering the structural features of these polymers. Typically, taking the spectrum of PPOP-1 for example, two major resonances at 127 and $140 \mathrm{ppm}$ are assigned to the unsubstituted phenyl carbon atoms and the substituted aromatic carbon atoms, respectively. The resonance at $46 \mathrm{ppm}$ can be ascribed to the tertiary carbon atoms that act as the linkage of two different benzene rings originated from the BPA and aldehyde monomers, respectively. The shoulder peak at $116 \mathrm{ppm}$ is related to the reacted ortho carbons of the hydroxy groups. The signal at $153 \mathrm{ppm}$ comes from the phenoxy carbons [25]. Unexpectedly, no obvious peak at $20 \mathrm{ppm}$ ascribed to the carbons of methyl groups from BPA molecules can be found in the spectrum of PPOP-1, which is in consistence with the results of FT-IR spectra, indicating the degradation of BPA catalyzed by TSA at high temperature $[29,30]$.

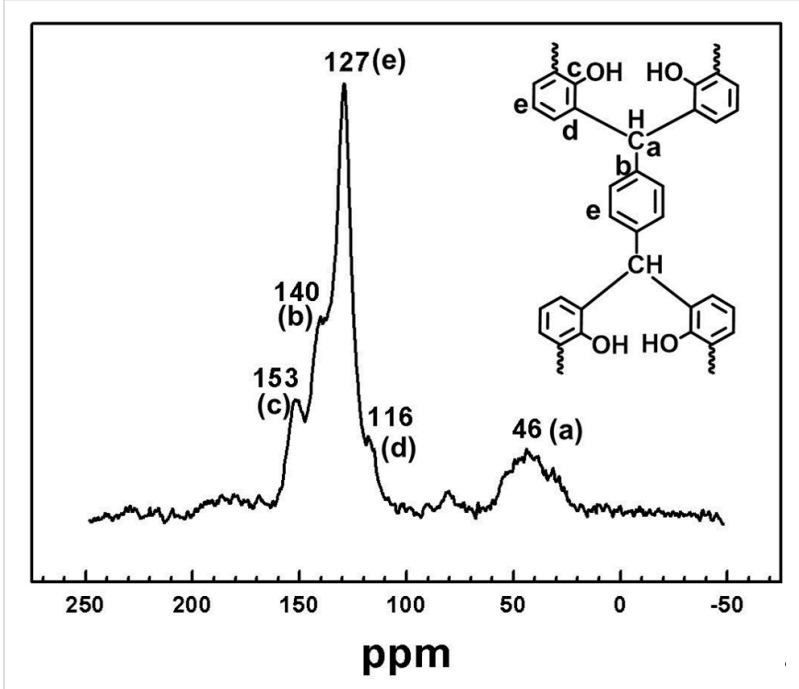

Figure 2: Solid-state ${ }^{13} \mathrm{C}$ CP/MAS NMR spectrum of PPOP-1 recorded at the MAS rate of $5 \mathrm{kHz}$. 
Nitrogen sorption measurements were employed to evaluate the porosity of the obtained polymers. The nitrogen adsorption-desorption isotherms of PPOP-1-PPOP-3 are similar to each other (Figure 3a). All of the isotherms show a high gas uptake at relative pressure $\left(P / P_{0}\right)$ less than 0.02 , indicating that the materials are microporous. Meanwhile, a nitrogen condensation step could be found for all the polymers at $P / P_{0}$ above 0.90 , which is an indication of characteristic macroporosity that might correspond to interparticular voids associated with the pack of small particles of about $4 \mu \mathrm{m}$ adhered to the external surface of spherical particles (Supporting Information File 1, Figure S5). The BET specific surface area values are calculated in the relative pressure range $P / P_{0}=0.01-0.10$ for the microporous materials [31] for PPOPs (Supporting Information File 1, Figure S6). PPOP-2 possesses the highest BET surface area value calculated as $920 \mathrm{~m}^{2} \mathrm{~g}^{-1}$. According to the obtained values summarized in Table 1 , both total pore volume $\left(0.36 \mathrm{~cm}^{3} \mathrm{~g}^{-1}\right)$ determined at $P / P_{0}=0.95$ and micropore volume $\left(0.18 \mathrm{~cm}^{3} \mathrm{~g}^{-1}\right)$ calculated using the $t$-plot method of PPOP-1 are smaller than those of PPOP-2 and PPOP-3. The difference between the pore volumes and BET specific surface area results of PPOPs may be related to the monomer strut length. With the shortest linker of M1, PPOP-1 possesses the lowest pore volume and BET specific surface area. As for PPOP-3, using M3 as a monomer may induce a depression of polymerization degree owing to its stereo-hindrance effect, which might be responsible for its lower BET surface area value $\left(880 \mathrm{~m}^{2} \mathrm{~g}^{-1}\right)$ and micropore volume $\left(0.20 \mathrm{~cm}^{3} \mathrm{~g}^{-1}\right)$ than that of PPOP-2 using M2 as the monomer. However, it is noteworthy that when the reaction is conducted between $\mathbf{M 1}$ and phenol selected as the substitution of BPA, a new material is obtained with a BET surface area value calculated as $470 \mathrm{~m}^{2} \mathrm{~g}^{-1}$ (Supporting Information File 1, Figure S7), which is a indication of the fact that pyrolysis of BPA might result in some new porous structure in situ, leading to an increase in BET surface area value. The PSD profiles calculated using original DFT are shown in Figure 3b. All of the materials exhibit a similar PSD profile with a maximum peak at $0.59 \mathrm{~nm}$ and several smaller peaks between 0.6 and $2.0 \mathrm{~nm}$, indicating that PPOPs are microporous. The pore size for PPOPs and the total pore volume for PPOP-2 and PPOP-3 do not show any obvious difference with increasing monomer strut length, which may be attributed to the random penetration and space-filling within the fragments of the extended repeating units [16].
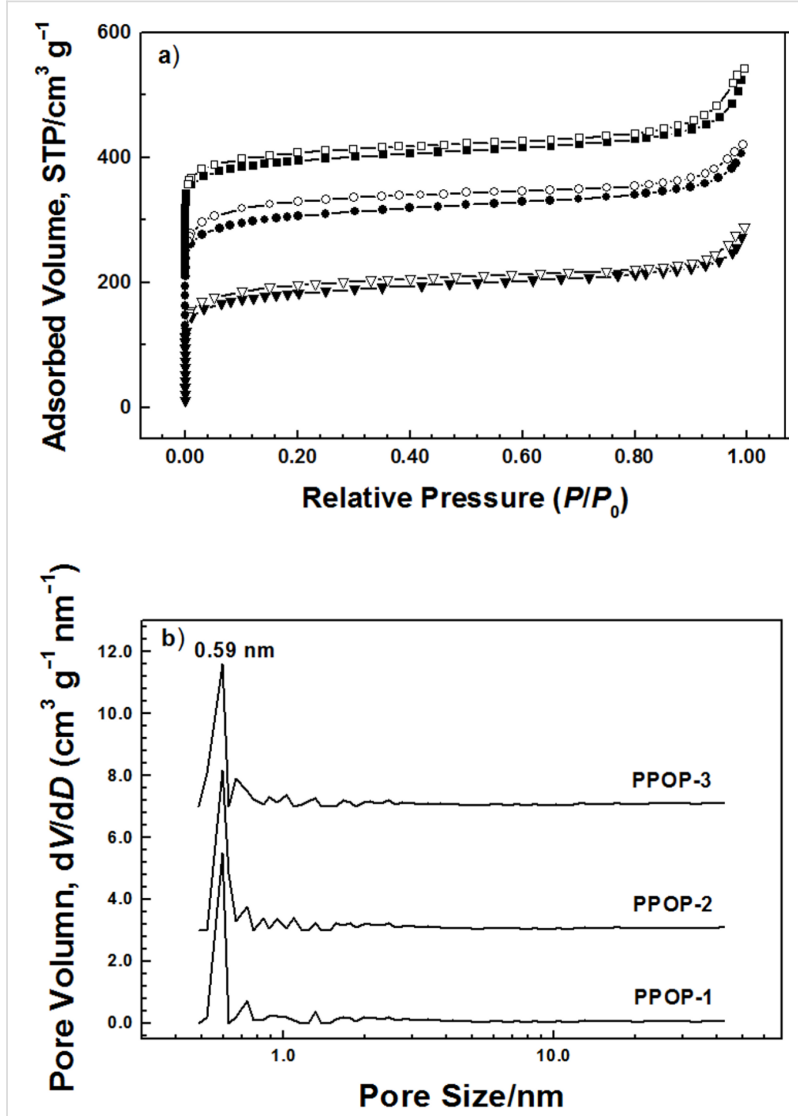

Figure 3: (a) Nitrogen adsorption-desorption isotherms of PPOP-1 (downtriangle), PPOP-2 (circle), and PPOP-3 (square) at $77 \mathrm{~K}$. The isotherms have been offset by $100 \mathrm{~cm}^{3} \mathrm{~g}^{-1}$ for PPOP-2 and $200 \mathrm{~cm}^{3} \mathrm{~g}^{-1}$ for PPOP-3 for the purpose of clarity, respectively. (b) PSD profiles calculated by the original DFT method. The PSD profiles of PPOP-2 and PPOP-3 have been offset by 3 and 6 units for the purpose of clarity, respectively.

The gas uptake capacities for carbon dioxide, hydrogen, and methane of the polymers are investigated by gravimetric methods and listed in Table 2. The hydrogen storage capacities for PPOPs vary between 1.08 and $1.28 \mathrm{wt} \%$ at $77 \mathrm{~K}$ and 1.0 bar (Figure 4a) and PPOP-3 possesses the highest hydrogen uptake,

Table 1: Porosity properties of PPOP-1-PPOP-3

\begin{tabular}{llll} 
polymer & $S_{\text {BET }}\left(\mathrm{m}^{2} \mathrm{~g}^{-1}\right)^{\mathrm{a}}$ & $V_{\text {total }}\left(\mathrm{cm}^{3} \mathrm{~g}^{-1}\right)^{\mathrm{b}}$ & $V_{\text {micro }}\left(\mathrm{cm}^{3} \mathrm{~g}^{-1}\right)^{\mathrm{c}}$ \\
\hline PPOP-1 & 720 & 0.36 & 0.18 \\
PPOP-2 & 920 & 0.41 & 0.21 \\
PPOP-3 & 880 & 0.41 & 0.20 \\
\hline
\end{tabular}

a Surface area calculated from the nitrogen adsorption isotherm using the BET method in the relative pressure $\left(P / P_{0}\right)$ range from 0.01 to 0.10 .

bTotal pore volume at $P / P_{0}=0.95$. cMicropore volume calculated from nitrogen adsorption isotherm using the $t$-plot method. 
Table 2: Gas adsorption uptake of PPOP-1-PPOP-3

\begin{tabular}{|c|c|c|c|c|}
\hline \multirow[t]{2}{*}{ polymer } & \multirow[t]{2}{*}{$\mathrm{H}_{2}$ uptake $(\text { wt } \%)^{\mathrm{a}}$} & \multirow[t]{2}{*}{$\mathrm{CH}_{4}$ uptake $(\text { wt } \%)^{\mathrm{b}}$} & \multicolumn{2}{|c|}{$\mathrm{CO}_{2}$ uptake $(\text { wt } \%)^{\mathrm{C}}$} \\
\hline & & & $273 \mathrm{~K}$ & $298 \mathrm{~K}$ \\
\hline PPOP-1 & 1.14 & 4.01 & 13.2 & 9.1 \\
\hline PPOP-2 & 1.08 & 4.29 & 14.6 & 8.2 \\
\hline PPOP-3 & 1.28 & 3.24 & 15.0 & 8.8 \\
\hline
\end{tabular}

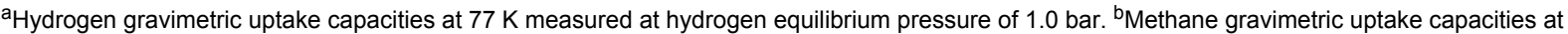
$273 \mathrm{~K}$ measured at a pressure at 1.0 bar. ${ }^{\circ}$ Carbon dioxide gravimetric uptake capacities at 1.0 bar measured at 273 and $298 \mathrm{~K}$, respectively.

which may be on account of the fact that there is much more ultramicropores in PPOP-3 that are appropriate for hydrogen rather than nitrogen [32]. The methane gravimetric uptake for the materials was measured at $273 \mathrm{~K}$ and 1.0 bar. PPOPs exhibit a methane storage capacity varying between 4.29 and $3.24 \mathrm{wt} \%$ (Figure $4 \mathrm{~d}$ ), which is higher than that of the reported mesoporous polymeric organic frameworks (mesoPOF)s [23]. PPOP-2 with the largest BET surface area and micropore volume shows the highest methane uptake. However, PPOP-3 possesses a smaller methane storage capacity than PPOP-1 that is known for its lowest BET surface area and total pore volume and micropore volume, which may arise from the fact that inter- actions between the accessible surface area, micropore volume, and pore topology contribute predominantly to methane storage capacity in porous material $[33,34]$. The carbon dioxide adsorption isotherms for PPOPs are collected at 273 and $298 \mathrm{~K}$, respectively (Figure 4b,c). PPOP-3 possesses a largest carbon dioxide adsorption capacity up to $15.0 \mathrm{wt} \%(273 \mathrm{~K})$ and $8.8 \mathrm{wt}$ $\%(298 \mathrm{~K})$ at $1.0 \mathrm{bar}$, which is larger than that of the reported work [35]. As reported that apparent surface area is not the only crucial factor that influences the amount of adsorbed $\mathrm{CO}_{2}$, whereas the uptake capacity is more depended on porosity characteristic such as pore size in the networks [36,37]. Specially, the smallest pores contribute most to the $\mathrm{CO}_{2}$ uptake
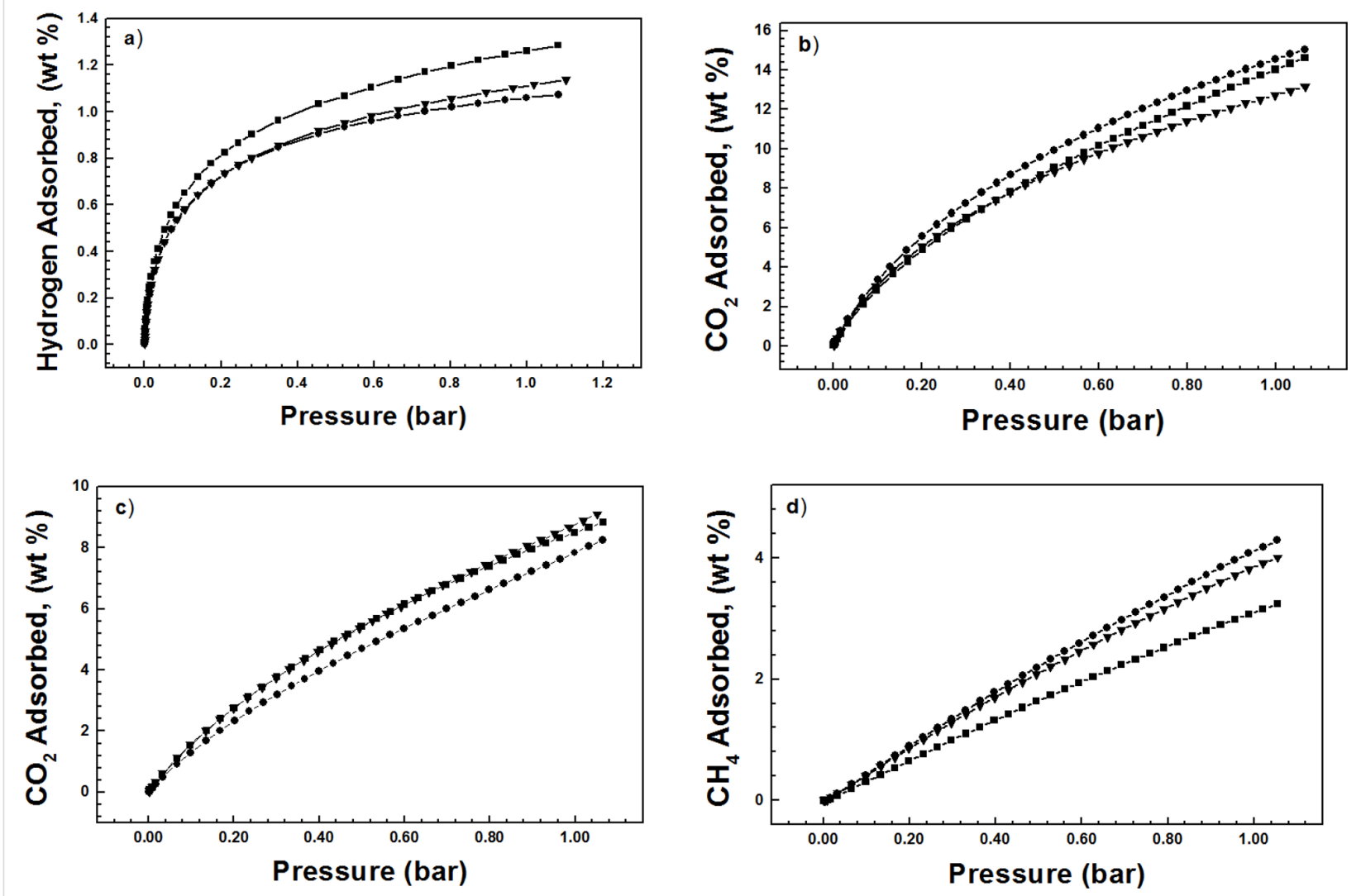

Figure 4: Gravimetric gas adsorption isotherms for PPOP-1 (downtriangle), PPOP-2 (circle), and PPOP-3 (square) (a) hydrogen at 77 K, (b) carbon dioxide at $273 \mathrm{~K}$, (c) carbon dioxide at $298 \mathrm{~K}$, and (d) methane at $273 \mathrm{~K}$. 
at low pressure [36]. Hence, PPOP-3 with a smaller pore size located at $0.68 \mathrm{~nm}$ that is different from the other two polymers in Figure $3 \mathrm{~b}$ is probably the best candidate for $\mathrm{CO}_{2}$ capture. The high carbon dioxide uptake capacity for PPOPs may correspond to the large amount of the hydroxy groups in the PPOPs through the formation of $\mathrm{O}=\mathrm{C}=\mathrm{O}\left(\delta^{-}\right) \ldots \mathrm{H}\left(\delta^{+}\right)-\mathrm{O}$ hydrogen bonds that are enhanced by weak supramolecular interactions with $\mathrm{C}-\mathrm{H}$ atoms on the aromatic rings of the polymers [38]. The isosteric heat of adsorption for carbon dioxide is calculated from adsorption data collected at 273 and $298 \mathrm{~K}$ using a virial method and the Clausius-Clapeyron equation [39] (Supporting Information File 1, Figure S8). The typical heats of absorption $Q_{\mathrm{st}}$ for the PPOPs are measured in the range of about $21.6-24.3 \mathrm{~kJ} \mathrm{~mol}^{-1}$ (Figure 5), which are in accordance with the report data [40], indicating that the adsorption of $\mathrm{CO}_{2}$ is mainly physical adsorption. Unusually, PPOP-2 and PPOP-3 show an increase in the $Q_{\text {st }}$ value with increased $\mathrm{CO}_{2}$ loading, which is likely induced by synergic interactions between carbon dioxide molecules $[41,42]$.

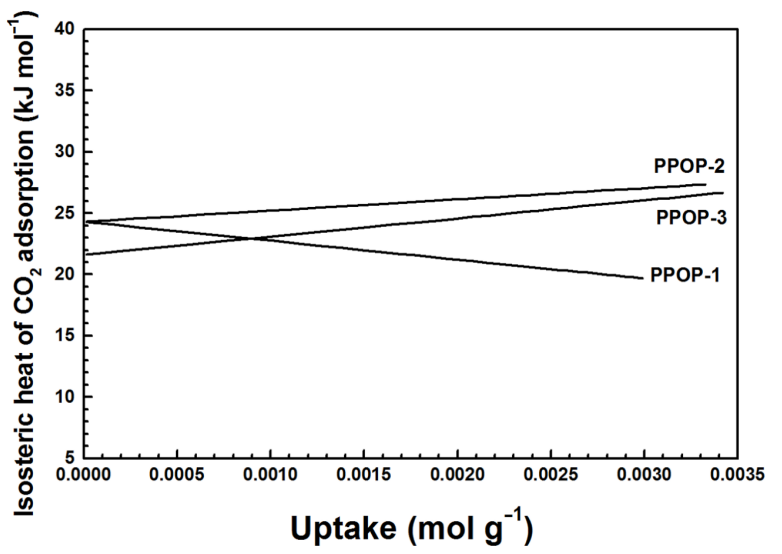

Figure 5: Variation of isosteric heat of adsorption with amount of adsorbed $\mathrm{CO}_{2}$ in PPOP-1, PPOP-2, and PPOP-3

\section{Conclusion}

In conclusion, we have developed a novel approach to porous organic polymers from BPA using the traditional Bakelite-type chemistry. The prepared polymers possess high specific surface area values up to $920 \mathrm{~m}^{2} \mathrm{~g}^{-1}$, with a high carbon dioxide uptake of up to $15.0 \mathrm{wt} \%$ at $273 \mathrm{~K}$ and 1.0 bar. The materials also exhibit hydrogen uptake properties measured as $1.28 \mathrm{wt} \%(77 \mathrm{~K})$ at 1.0 bar while the highest methane storage capacity is $4.29 \mathrm{wt} \%(273 \mathrm{~K})$ at 1.0 bar. These gas adsorption properties and high BET specific surface area may make the PPOPs appropriate candidates for materials for gas adsorption and storage.

\section{Experimental}

\section{Preparation of PPOPs}

A mixture of BPA (50.0 mg, $0.22 \mathrm{mmol})$, terephthalaldehyde (59.0 $\mathrm{mg}, 0.44 \mathrm{mmol})$, and $p$-toluenesulfonic acid $(0.5 \mathrm{~g})$ was suspended in $o$-dichlorobenzene $(8.0 \mathrm{~mL})$ in a glass tube. After ultrasonication for $0.5 \mathrm{~h}$, the mixture was degassed by at least three freeze-pump-thaw cycles. The tube was frozen at $77 \mathrm{~K}$ (liquid nitrogen bath) and evacuated to high vacuum and flamesealed. After $180^{\circ} \mathrm{C}$ for $72 \mathrm{~h}$, the reaction mixture gave a solid product (denoted as PPOP-1). After cooled to room temperature, the solid was filtrated and washed with acetone, dichloromethane, and ethanol, subsequently. Further purification of the polymer was carried out by Soxhlet extraction with water, ethanol, and dichloromethane for $24 \mathrm{~h}$ to give the final product with a yield of $87.5 \%$, which was dried in vacuo at $120{ }^{\circ} \mathrm{C}$ for more than $12 \mathrm{~h}$.

Similar to the preparation of PPOP-1, 4,4'-biphenyldicarboxaldehyde (M2) and 1,3,5-tri(4-formylphenyl)benzene (M3) were used to afford PPOP-2 and PPOP-3, with yield of $85 \%$ and $80 \%$, respectively.

\section{Supporting Information}

\section{Supporting Information File 1}

Experimental, instruments section, SEM images, data of TGA, FTIR and BET surface area, virial analysis of the adsorption data for $\mathrm{CO}_{2}$ and NMR spectra. [http://www.beilstein-journals.org/bjoc/content/ supplementary/1860-5397-13-211-S1.pdf]

\section{Acknowledgements}

The financial support of the National Natural Science Foundation of China (Grant no. 21574032) and the Ministry of Science and Technology of China (Grant no. 2014CB932200) is acknowledged.

\section{References}

1. Yaghi, O. M.; Li, G.; Li, H. Nature 1995, 378, 703-706. doi:10.1038/378703a0

2. Yaghi, O. M.; Li, H. J. Am. Chem. Soc. 1995, 117, 10401-10402. doi:10.1021/ja00146a033

3. Côté, A. P.; Benin, A. I.; Ockwig, N. W.; O'Keeffe, M.; Matzger, A. J.; Yaghi, O. M. Science 2005, 310, 1166-1170. doi:10.1126/science.112041

4. Wan, S.; Guo, J.; Kim, J.; Ihee, H.; Jiang, D. Angew. Chem., Int. Ed. 2008, 47, 8826-8830. doi:10.1002/anie.200803826

5. Palkovits, R.; Antonietti, M.; Kuhn, P.; Thomas, A.; Schüth, F. Angew. Chem., Int. Ed. 2009, 48, 6909-6912. doi:10.1002/anie.200902009

6. Liu, J.; Chen, Q.; Sun, Y.-N.; Xu, M.-Y.; Liu, W.; Han, B.-H. RSC Adv. 2016, 6, 48543-48549. doi:10.1039/c6ra04515d 
7. Du, X.; Sun, Y.; Tan, B.; Teng, Q.; Yao, X.; Su, C.; Wang, W. Chem. Commun. 2010, 46, 970-972. doi:10.1039/B920113K

8. Chan-Thaw, C. E.; Villa, A.; Katekomol, P.; Su, D.; Thomas, A.; Prati, L. Nano Lett. 2010, 10, 537-541. doi:10.1021/nl904082k

9. Kaur, P.; Hupp, J. T.; Nguyen, S. T. ACS Catal. 2011, 1, 819-835. doi:10.1021/cs200131g

10. Ghanem, B. S.; McKeown, N. B.; Budd, P. M.; Selbie, J. D.; Fritsch, D. Adv. Mater. 2008, 20, 2766-2771. doi:10.1002/adma.200702400

11. Du, N.; Park, H. B.; Robertson, G. P.; Dal-Cin, M. M.; Visser, T.; Scoles, L.; Guiver, M. D. Nat. Mater. 2011, 10, 372-375. doi:10.1038/nmat2989

12. Wood, C. D.; Tan, B.; Trewin, A.; Niu, H.; Bradshaw, D.; Rosseinsky, M. J.; Khimyak, Y. Z.; Campbell, N. L.; Kirk, R.; Stöckel, E.; Cooper, A. I. Chem. Mater. 2007, 19, 2034-2048. doi:10.1021/cm070356a

13. Hu, X.-M.; Chen, Q.; Zhao, Y.-C.; Laursen, B. W.; Han, B.-H. Microporous Mesoporous Mater. 2016, 224, 129-134. doi:10.1016/j.micromeso.2015.11.046

14. McKeown, N. B.; Gahnem, B.; Msayib, K. J.; Budd, P. M.; Tattershall, C. E.; Mahmood, K.; Tan, S.; Book, D.; Langmi, H. W.; Walton, A. Angew. Chem., Int. Ed. 2006, 45, 1804-1807. doi:10.1002/anie.200504241

15. Wu, D.; Xu, F.; Sun, B.; Fu, R.; He, H.; Matyjaszewski, K. Chem. Rev. 2012, 112, 3959-4015. doi:10.1021/cr200440z

16. Jiang, J.-X.; Su, F.; Trewin, A.; Wood, C. D.; Niu, H.; Jones, J. T. A.; Khimyak, Y. Z.; Cooper, A. I. J. Am. Chem. Soc. 2008, 130, 7710-7720. doi:10.1021/ja8010176

17. Weber, J.; Thomas, A. J. Am. Chem. Soc. 2008, 130, 6334-6335. doi:10.1021/ja801691x

18. Liu, X.; Xu, Y.; Jiang, D. J. Am. Chem. Soc. 2012, 134, 8738-8741. doi:10.1021/ja303448r

19. Kuhn, P.; Antonietti, M.; Thomas, A. Angew. Chem., Int. Ed. 2008, 47, 3450-3453. doi:10.1002/anie.200705710

20. Zhao, Y.-C.; Cheng, Q.-Y.; Zhou, D.; Wang, T.; Han, B.-H. J. Mater. Chem. 2012, 22, 11509-11514. doi:10.1039/C2JM31187A

21. Zhao, Y.-C.; Wang, T.; Zhang, L.-M.; Cui, Y.; Han, B.-H. ACS Appl. Mater. Interfaces 2012, 4, 6975-6981. doi:10.1021/am302163p

22. Wang, T.; Zhao, Y.-C.; Luo, M.; Zhang, L.-M.; Cui, Y.; Zhang, C.-S.; Han, B.-H. Polymer 2015, 60, 26-31. doi:10.1016/j.polymer.2014.12.072

23. Katsoulidis, A. P.; Kanatzidis, M. G. Chem. Mater. 2012, 24, 471-479. doi:10.1021/cm202578k

24. Katsoulidis, A. P.; He, J.; Kanatzidis, M. G. Chem. Mater. 2012, 24, 1937-1943. doi:10.1021/cm300696g

25. Katsoulidis, A. P.; Kanatzidis, M. G. Chem. Mater. 2011, 23, 1818-1824. doi:10.1021/cm103206x

26. Wisser, F. M.; Eckhardt, K.; Wisser, D.; Böhlmann, W.; Grothe, J.; Brunner, E.; Kaskel, S. Macromolecules 2014, 47, 4210-4216. doi:10.1021/ma500512j

27. Rose, M.; Klein, N.; Senkovska, I.; Schrage, C.; Wollmann, P.; Böhlmann, W.; Böhringer, B.; Fichtner, S.; Kaskel, S. J. Mater. Chem. 2011, 21, 711-716. doi:10.1039/C0JM02998J

28. Jang, B. N.; Wilkie, C. A. Polym. Degrad. Stab. 2004, 86, 419-430. doi:10.1016/j.polymdegradstab.2004.05.009

29. Hunter, S. E.; Felczak, C. A.; Savage, P. E. Green Chem. 2004, 6, 222-226. doi:10.1039/B313509H

30. Hunter, S. E.; Savage, P. E. J. Org. Chem. 2004, 69, 4724-4731. doi:10.1021/j00356964
31. Dawson, R.; Laybourn, A.; Clowes, R.; Khimyak, Y. Z.; Adams, D. J.; Cooper, A. I. Macromolecules 2009, 42, 8809-8816. doi:10.1021/ma901801s

32. Zhang, B.; Wang, Z. Chem. Commun. 2009, 5027-5029. doi:10.1039/B909424E

33. Makal, T. A.; Li, J.-R.; Lu, W.; Zhou, H.-C. Chem. Soc. Rev. 2012, 41, 7761-7779. doi:10.1039/C2CS35251F

34. Düren, T.; Sarkisov, L.; Yaghi, O. M.; Snurr, R. Q. Langmuir 2004, 20, 2683-2689. doi:10.1021/la0355500

35. Dawson, R.; Stevens, L. A.; Drage, T. C.; Snape, C. E.; Smith, M. W.; Adams, D. J.; Cooper, A. I. J. Am. Chem. Soc. 2012, 134, 10741-10744. doi:10.1021/ja301926h

36. Dawson, R.; Stöckel, E.; Holst, J. R.; Adams, D. J.; Cooper, A. I. Energy Environ. Sci. 2011, 4, 4239-4245. doi:10.1039/C1EE01971F

37. Wahby, A.; Ramos-Fernández, J. M.; Martínez-Escandell, M.; Sepúlveda-Escribano, A.; Silvestre-Albero, J.; Rodríguez-Reinoso, F. ChemSusChem 2010, 3, 974-981. doi:10.1002/cssc.201000083

38. Yang, S.; Sun, J.; Ramirez-Cuesta, A. J.; Callear, S. K.; David, W. I. F.; Anderson, D. P.; Newby, R.; Blake, A. J.; Parker, J. E.; Tang, C. C.; Schröder, M. Nat. Chem. 2012, 4, 887-894. doi:10.1038/nchem.1457

39. Sumida, K.; Rogow, D. L.; Mason, J. A.; McDonald, T. M.; Bloch, E. D.; Herm, Z. R.; Bae, T.-H.; Long, J. R. Chem. Rev. 2012, 112, 724-781. doi:10.1021/cr2003272

40. Dunne, J. A.; Mariwala, R.; Rao, M.; Sircar, S.; Gorte, R. J.; Myers, A. L. Langmuir 1996, 12, 5888-5895. doi:10.1021/la960495z

41. Furukawa, H.; Yaghi, O. M. J. Am. Chem. Soc. 2009, 131, 8875-8883. doi:10.1021/ja9015765

42. Llewellyn, P. L.; Maurin, G. C. R. Chim. 2005, 8, 283-302. doi:10.1016/j.crci.2004.11.004

\section{License and Terms}

This is an Open Access article under the terms of the Creative Commons Attribution License (http://creativecommons.org/licenses/by/4.0), which permits unrestricted use, distribution, and reproduction in any medium, provided the original work is properly cited.

The license is subject to the Beilstein Journal of Organic Chemistry terms and conditions:

(http://www.beilstein-journals.org/bjoc)

The definitive version of this article is the electronic one which can be found at: doi:10.3762/bjoc.13.211 